\title{
绿 \\ PCA BASED Image Fusion of Face and Iris Biometric Features
}

\author{
S. Anu H Nair, P.Aruna \& M.Vadivukarassi \\ CSE Department, Annamalai University, Annamalai Nagar, Chidambaram, Tamil Nadu, India
}

\begin{abstract}
This paper is used to implement feature level fusion for the extracted images of the different biometric features. The biometric features used here are face and iris. Discrete Wavelet Transform (DWT) and Discrete Cosine Transform (DCT) are used for feature extraction of face and iris independently and compared. The features of face and iris are fused by PCA fusion technique. Experimental results on ORL(Olivetti Research Laboratory) face database and CASIA (Chinese Academy of Sciences Institute of Automation)iris database are verified. MATLAB software is used to implement this multimodal biometric system.
\end{abstract}

Keywords: discrete cosine transform, discrete wavelet transform, feature extraction, fusion.

\section{INTRODUCTION}

The term 'Biometric' highlights the use of biological, physical or behavioural traits of a person to identity or verify his/her identity. A biometric is defined as "life measure" and biometric technology uses images of human body parts, captured through cameras and scanning images. Multimodal biometrics refers to the combination of two or more biometric modalities.

\section{A. Related work}

Chaunte W. Lacewell et al.,[1] conducted survey on techniques of image fusion to obtain the most accurate information. To produce accurate fused image, DWT was used for feature extraction and Genetic algorithm is used to get more optimized combined image. Jun-Ying Gan et al., [2] proposed an efficient algorithm for fusion and recognition of face and iris feature based on wavelet transform. DWT method was used to reduce the dimension, to eliminate the noise, to save the space and to improve the efficiency

S. Arun Vivek, J. Araind et al.,[3] described about the study of multimodal biometric system. The feature extraction techniques were used for three modalities. The extracted information from each modality was stored as template.

Jun -Ying Gan et al., [5] suggested the research on Face and Iris feature recognition based on
2DDCT and Kernel Fisher Discriminant Analysis method for feature fusion. This new approach was supplied for multimodal biometric identification.

Ashish Mishra et al.,[7] explained the different types of multimodal biometric system, different decision fusion techniques used in the system. This study discussed the feasibility and advantages over unimodal biometric system. Sheetal Choudhary et al.,[11]introduced a multimodal biometric recognition system integrating palmprint, fingerprint and face based on score level fusion. The feature vectors were extracted independently from the pre-processed images of palmprint, fingerprint and face.

Li Bai et al., [10] proposed to improve the accuracy of personal identification, when only single sample was registered as template, by integrating multiple handbased biometrics, i.e. palmprint and finger-knuckleprint. Lillin Shen et al., [10] had aims to improve the accuracy of personal identification, when only single sample was registered as template, by integrating multiple hand-based biometrics. To make fusion much easier, the same feature,called fusion code, and decision level fusion strategy were used. Two fusion cases face \& palmprint and FKP \& palmprint were taken as examples to verify the effectiveness.

Gemma Piella et at .,[13] presented a new approach for assessing quality in image fusion and compared different image fusion methods or to find the best parameters for a given fusion algorithm. Deepak Kumar Sahul et al.,[14] proposed PCA based image fusion and also focuses on image fusion algorithm based on wavelet transform to improve resolution of the images in which two images to be fused are firstly decomposed into subimages with different frequency and then the information fusion is performed and finally these subimages are reconstructed into result image with plentiful information. Manjusha Deshmukh et al.,[15] introduced a literature review on some of the image fusion techniques for image fusion like, primitive fusion (Averaging Method, Select Maximum, and Select Minimum), Discrete Wavelet transform based fusion, Principal component analysis (PCA). 


\section{FEATURE EXTRACTION OF FACE AND IRIS}

The major steps involved in a general Biometric system are; image acquisition, pre-processing, feature extraction and feature fusion. The Single image of face and iris from the database are the input for the preprocessing method.

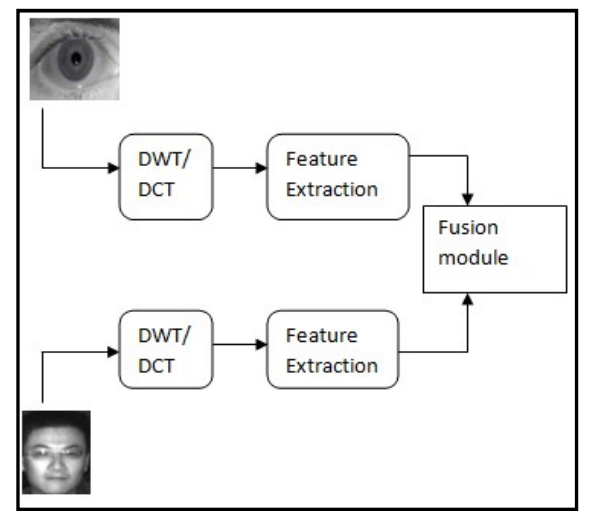

Fig 1 Block diagram of fusion of face and iris

In feature extraction, morphological or textual attributes are elicited. This uniquely represents the input image. The techniques used for feature extraction are Discrete Cosine Transform (DCT), Discrete Wavelet Transform (DWT). Feature extraction involves simplifying the amount of resources required to describe a large set of data accurately.

\section{A. Face feature extraction}

\section{DWT}

For 2D discrete wavelet transform (DWT), an image is represented in terms of translations and dilations of a scaling function and a wavelet functions. After one level of 2D decomposition, an image is divided into four sub-bands: LL (Low-Low), which is generated by the approximation coefficients; LH (LowHigh), HL (High-Low), and HH (High-High), which are generated by the detail coefficients, as shown in Fig. 2.
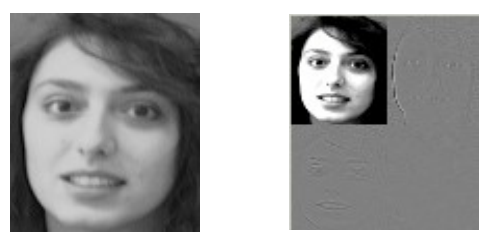

Fig 2 Original Face image, and 1-level 2D-DWT

DWT can be mathematically expressed by (1):

$\operatorname{DWTx}(n)=\left\{\begin{array}{l}\mathrm{d}_{\mathrm{j}, \mathrm{k}}=\sum \mathrm{x}(\mathrm{n}) \mathrm{h}\left(\mathrm{n}-2^{\mathrm{j}} \mathrm{k}\right) \\ \mathrm{a}_{\mathrm{j}, \mathrm{k}}=\sum \mathrm{x}(\mathrm{n}) \mathrm{g}\left(\mathrm{n}-2^{\mathrm{j}} \mathrm{k}\right)\end{array}\right.$

The coefficients $d_{j, k}$ refer to the detail components in signal $x(n)$ and correspond to the wavelet function, whereas $a_{j, k}$ refers to the approximation components in the signal. The functions $h(n)$ and $g(n)$ in the equation represent the coefficients of the high-pass and low-pass filters respectively, while parameters $j$ and $k$ refer to wavelet scale and translation factors.

\section{2. $D C T$}

Face images have high correlation and redundant information which cause computational burden in terms of processing speed and memory utilization. The DCT transforms images from the spatial domain to the frequency domain. This reduces data volume without sacrificing too much image quality.

The general equation for the DCT of an NxM image $f(x, y)$ is defined by the following equation:

$$
\text { N-1 N-1 }
$$

$\mathrm{F}(\mathrm{u}, \mathrm{v})=\alpha(\mathrm{u}) \alpha(\mathrm{v}) \sum \sum \cos [\pi \cdot \mathrm{u} / 2 \mathrm{~N}(2 \mathrm{x}+1)]$.

$$
\mathrm{x}=0 \quad \mathrm{y}=0
$$

$$
\cos [\pi \cdot u / 2 M(2 y+1)] f(x, y)
$$
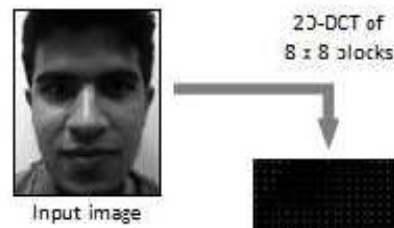

nput image
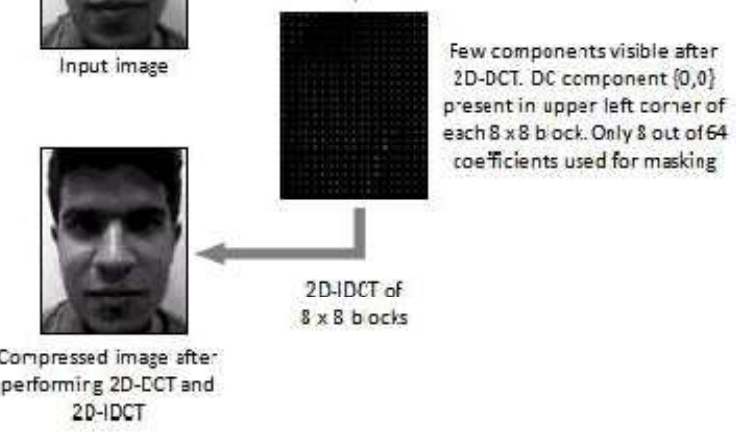

Fig. 3 2D-DCT computation of face image

Where $f(x, y)$ is the intensity of the pixel in row $x$ and

$(u), \alpha(v) ₹ \begin{cases}\sqrt{1 / \mathbb{N}} & \text { for } \mathrm{u}, \mathrm{v}=0 \\ \sqrt{2} / \mathbb{N} & \text { for } \mathrm{u}, \mathrm{v} \neq 0\end{cases}$

$u=0,1, \ldots ., N-1$ and

$v=0,1, \ldots, M-1$ and the functions $\alpha(u), \alpha(v)$ are also defined.

\section{Face extraction}

The process of face extraction involves identifying the person based on facial features. Hence it is necessary to extract the features from a given image. Directional binary code(DBC) is applied on LL sub band to encode the directional edge information. It captures the spatial relationship between any pair of neighbourhood pixels in a local region along a given direction. It reflects the image local feature. It extracts more spatial information than LBP.

Let $\mathrm{Z}_{\mathrm{i}, \mathrm{j}}$ be a point in a cell. 
$\mathrm{I}_{(\alpha, \mathrm{d})}\left(\mathrm{Z}_{\mathrm{i}, \mathrm{j}}\right)=\mathrm{I}\left(\mathrm{Z}_{\mathrm{i}, \mathrm{j}}\right)-\mathrm{I}\left(\mathrm{Z}_{\mathrm{i}, \mathrm{j}-\mathrm{d}}\right)$

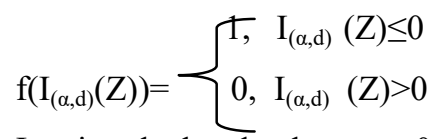

$\mathrm{I}_{(\alpha, \mathrm{d})}$ is calculated, where $\alpha=0^{0}, 45^{0}, 90^{0}, 135^{0}$

$\mathrm{d}$ is the distance between the given point and its neighbouring point.

\section{B. Iris feature extraction}

\section{Iris image pre-processing}

Iris is one of the most reliable and accurate biometric system. The iris is the opaque, colored portion of the eye that surrounds the pupil. Image pre-processing part is performed before extracting iris features. It is processed to localize the inner and outer boundary of iris in the captured iris image

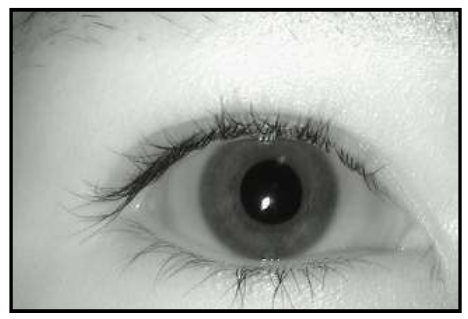

Fig 4 Input Image of iris

The localized iris region is transformed into polar coordinate to compensate iris image deformation due to the variation of pupil size in the conditions of iris image.

In fact, the features of the iris are extracted based on the Polar Coordinate. An iris code is a binary matrix, representative of an individual's iris pattern. An iris code stored in a database is considered the template to which future iris codes are tested in order to determine similarity. The segmentation module locates the position of the iris within the image by isolating it from the sclera, pupil, eyelids, and eyelashes.

\section{2. Canny edge detection and localization}

Canny edge detection is used to create an edge map . The Canny method finds edges by looking for local maxima of the gradient of the iris image. The Canny edge detects strong and weak edges, and includes the weak edges in the output only if they are connected to strong edges. Here the boundary of an iris is located using parameters like centre coordinates $\mathrm{x}$ and $\mathrm{y}$, the radius $r$, which are related according to the following equation,

$$
x^{2}+y^{2}=r^{2}
$$

In performing the preceding edge detection step, the derivatives of the horizontal direction will detect the eyelids, and the vertical direction will detect the outer circular boundary of the iris. The radius of the iris image is determined and provided to the Hough transform. For better accuracy, the Hough transform is carried out initially for iris/sclera boundary and then for iris/pupil boundary.

\section{3. Hough transform}

The Hough transform is a feature extraction technique used in image analysis. It finds imperfect instances of objects within a certain class of shapes by a voting procedure. This voting procedure is carried out in a parameter space, from which object candidates are obtained as local maxima in the accumulator space that is explicitly constructed by the algorithm for computing the Hough transform. Canny edge detection is used to build the edges in horizontal direction and then the Parabolic Hough transform is applied on it to detect the eyelids, approximating the upper and lower eyelids with parabolic arcs. . If the maximum Hough space is below the threshold it indicates the non occlusion of eyelids. For isolating eyelashes it is very easy by utilizing thresholding. This is because they are darker while comparing with further elements in eye.

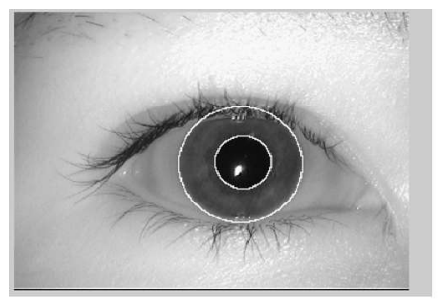

Fig 5 Inner and outer boundary of iris

\section{Normalization}

Once the segmentation module has estimated the iris's boundary, the normalization module transforms the iris texture from Cartesian to polar coordinates. The process called iris unwrapping, yields a rectangular entity that is used for subsequent processing. The normal Cartesian to polar transformation is recommended. This maps the entire pixels in the iris area into a pair of polar coordinates $(r, \theta)$, where $r$ and $\theta$ represent the intervals of [l $\left.\begin{array}{ll}0 & 1\end{array}\right]$ and $\left[\begin{array}{ll}0 & 2 \pi\end{array}\right]$. Normalization has advantages. It accounts for variations in pupil size due to changes in external illumination that will influence iris size. It ensures that the irises of different individuals are mapped onto a common image domain in spite of the variations in pupil size across subjects etc.

\section{Histogram equalization}

Histogram equalization is done on iris template to generate an image whose intensity covers the entire range of intensity levels. The normalized iris image has very low contrast and it could have a non-uniform brightness in different parts of the image due to the light applied at the acquisition time. This makes the iris texture seem to be with less contrast than it really is. The contrast enhancement of the image is accomplished by means of histogram equalization in order to use the full spectrum of gray levels and the textures are highlighted. Further, filtering operation can be applied to remove noisy components. 


\section{Iris code generation and Feature extraction}

Feature extraction is a key process where the two dimensional image is converted to a set of mathematical parameters. The iris contains important unique features, such as stripes, freckles, coronas, etc. These features are collectively referred to as the texture of the iris. These features were extracted using following algorithms.

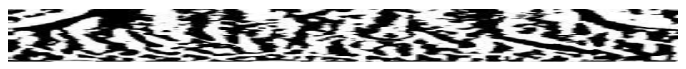

Fig 6 Iris code generation

\section{FUSION}

\section{A. Image fusion}

Image fusion is the process of combining relevant information from two or more images into a single image. The resulting image will be more informative than any of the input images. Fusion of face and iris is user friendly and well accepted system with better performance. The most important issue concerning image fusion is to determine how to combine the sensor images. In recent years, several image fusion techniques have been proposed

Feature level methods are the next stage of processing where image fusion will take place. Fusion at the feature level requires extraction of features from the input images. Features can be pixel intensities or edge and texture features. The Various kinds of features are considered depending on the nature of images and the application of the fused image. The features involve the extraction of feature primitives like edges, regions, shape, size, length or image segments, and features with similar intensity in the images to be fused from different types of images of the same geographic area. These features are later combined with the similar features present in the other input images through a predetermined selection process to form the final fused image. The feature level fusion should be easy. However, feature level fusion is difficult to achieve when the feature sets are derived from different algorithms and data sources .

\section{B. PCA based image fusion}

PCA based image fusion is implemented from the extracted images of face and iris. It is used for dimensionality reduction, feature enhancement, and image fusion. PCA transforms or projects the features from the original domain to a new domain (known as PCA domain).Here the features are arranged in the order of their variance. Fusion process is achieved in the PCA domain by retaining only those features that contain a significant amount of information. The main idea behind PCA is to determine the features that explain as much of the total variation in the data as possible with as few of these features as possible.

The computation of the PCA transformation matrix is based on the eigen value decomposition of the covariance matrix $\Sigma$ is defined as:

$$
\Sigma=\sum_{\mathrm{i}=1}^{\mathrm{N}^{2}}\left(\mathrm{X}_{\mathrm{i}}-\mathrm{m}\right)\left(\mathrm{X}_{\mathrm{i}}-\mathrm{m}\right)^{\mathrm{T}}
$$

where $\mathrm{X}$ is the $\mathrm{i}^{\text {th }}$ spectral signature, $\mathrm{m}$ denotes the mean spectral signature and $\mathrm{N}^{2}$ is the total number of spectral signatures. In order to find the new orthogonal axes of the PCA space, Eigen decomposition of the covariance matrix is $\sum$ performed

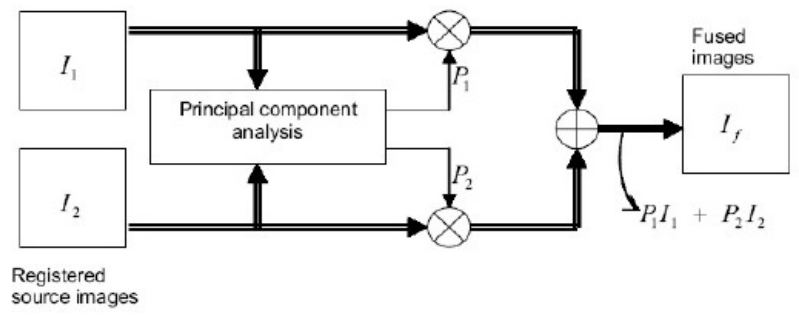

Fig 7 Information flow diagram in image fusion scheme employing PCA.

The eigen decomposition of the covariance matrix is given by

$\Sigma \mathrm{a}_{\mathrm{k}}=\lambda_{\mathrm{k}} \mathrm{a}_{\mathrm{k}}$

where $\lambda_{\mathrm{k}}$ denotes the $\mathrm{k}^{\text {th }}$ eigen value, $\mathrm{a}_{\mathrm{k}}$ denotes the corresponding eigenvector and $\mathrm{k}$ varies from 1 to 3 . The eigen values denote the amount of variance present in the corresponding eigen vectors. The eigenvectors form the axes of the PCA space, and they are orthogonal to each other. The eigen values are arranged in the decreasing order of the variance.

\section{EXPERIMENTAL RESULTS}

The multimodal biometric system is evaluated with the performance based on the ORL face database and CASIA iris database.

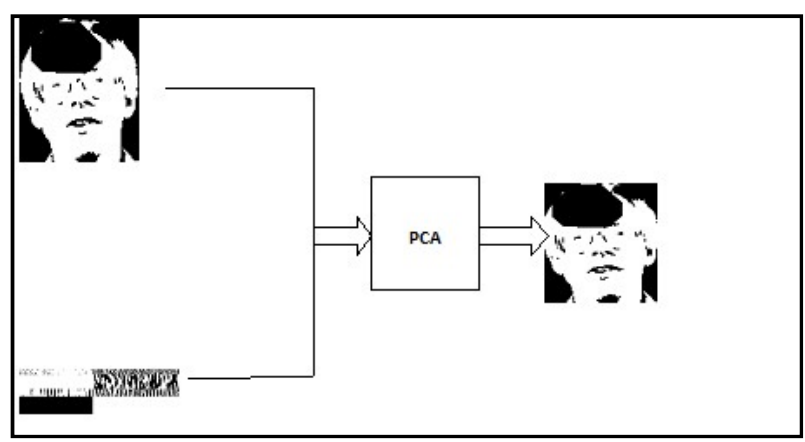

Fig 8 Fusion of images using PCA

The experimental results reveal the multimodal biometrics system is more reliable and precise than the single biometric approach. In Fig 8, the extracted images of face and iris are fused by the PCA image fusion method respectively

\section{A. Performance Evaluation}

Image Quality Measurement (IQM) is vital in the development of image processing algorithms such as 
enhancement, deblurring, denoising etc. as it can be used to evaluate their performances in terms of the quality of processed image.

The Mean Square Error (MSE) and the Peak Signal to Noise Ratio (PSNR) are the two error metrics used to compare image compression quality. The MSE represents the cumulative squared error between the compressed and the original image, whereas PSNR represents a measure of the peak error. To compute the PSNR, the block first calculates the mean-squared error using the following equation:

$$
\operatorname{MSE}=\frac{1}{M N} \sum_{i=0}^{M-1} \sum_{j=0}^{N-1}[I(i, j)-k(i, j)]^{2}
$$

In the previous equation, $\mathbf{M}$ and $\mathbf{N}$ are the number of rows and columns in the input images, respectively. Further, the block computes the PSNR using the following equation:

$$
\mathrm{PSNR}=10 \log _{10}\left(\frac{255^{2}}{M S E}\right)
$$

Normalized Cross-Correlation(NCC) is one of the methods used for template matching, a process used for finding incidences of a pattern or object within an image.

$$
\mathrm{NCC}=\frac{\sum_{(\mathrm{i}, \mathrm{j}) \square \mathrm{w}} \mathrm{I}_{\mathrm{l}}(\mathrm{i}, \mathrm{j}) \cdot \mathrm{I}_{2}(\mathrm{x}+\mathrm{i}, \mathrm{j}+\mathrm{j})}{\sqrt{\sum_{(\mathrm{i}, \mathrm{j}) \square \mathrm{w}} \mathrm{I}_{1}{ }^{2}(\mathrm{I}, \mathrm{j}) \cdot \sum_{(\mathrm{i}, \mathrm{j}) \square \mathrm{w}} \mathrm{I}_{2}{ }^{2}(\mathrm{x}+\mathrm{i}, \mathrm{y}+\mathrm{j})}}(14)
$$

TABLE-1 RESULTS OF PSNR, NCC, NAE

\begin{tabular}{|c|c|c|c|c|}
\hline \multirow{2}{*}{ Performance } & \multicolumn{2}{|c|}{ DWT } & \multicolumn{2}{c|}{ DCT } \\
\cline { 2 - 5 } & Face & Iris & Face & Iris \\
\hline PSNR(db) & 48.13 & 48.13 & 40.59 & 43.70 \\
\hline NCC & 0.424 & 0.465 & 0.003 & 0.003 \\
\hline NAE & 0.591 & 0.545 & 0.996 & 0.990 \\
\hline
\end{tabular}

Normalized Absolute Error (NAE) is the numerical difference between the original and reconstructed image. The large value of NAE means that image is of poor quality.NAE is defined as:-

$$
\mathrm{NAE}=\frac{\sum_{i=1, j=1}^{M N}\left|\mathrm{X}(\mathrm{i}, \mathrm{j})-\mathrm{X}^{\wedge}(\mathrm{i}, \mathrm{j})\right|}{\sum_{\mathrm{i}=1}^{M} \sum_{j=1}^{N}|\mathrm{X}(\mathrm{i}, \mathrm{j})|}
$$

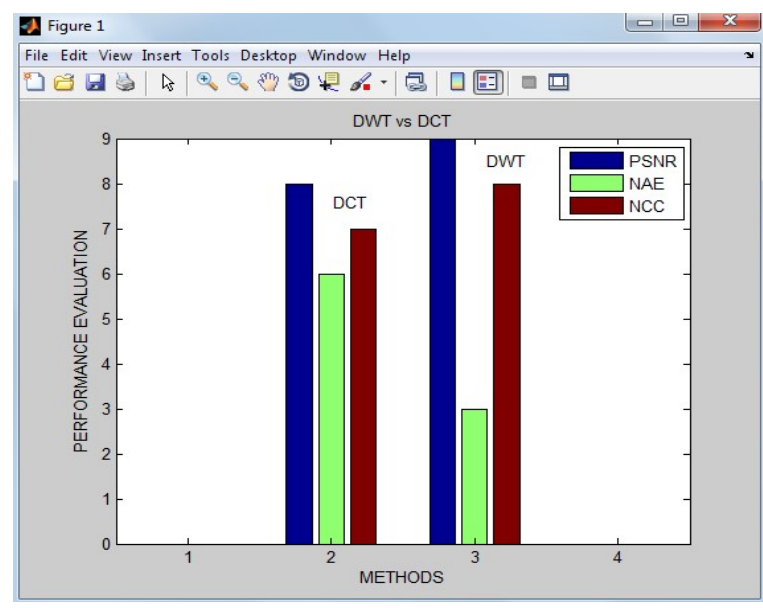

Fig 9 Comparison between DCT and DWT

Thus performances are analysed and the comparison results related to PSNR described in table1 clearly show that the advantages of DWT are better compared to the DCT. DWT has higher flexibility, higher compression ratio and higher PSNR value, compared to DCT. Therefore, DWT is chosen as the best transformation method.

The system was evaluated in MATLAB using an image database of face and iris. PSNR values of DWT images are higher than the DCimages of face and iris. So in feature level fusion, DWT feature extraction of face and iris are chosen as the best by comparing with the DCT feature extraction of face and iris respectively.

\section{CONCLUSION}

Multimodal Biometric Systems are expected to be more reliable. Based on the study of multimodal biometric system, the features of face and iris are extracted separately using DWT and DCT. The feature extraction of face and iris are very complex and non linear. These images are first decomposed for feature extraction and later the extracted images are fused. The experimental results show the face and iris biometric feature fusion has been implemented. The performance of DCT and DWT are evaluated using PSNR and DWT analysed as the best feature extraction technique. The fused image can be further used for watermarking and authentication purposes. 


\section{REFERENCES}

1. Chaunte W.Lacewell, ohamed Gebril, Ruben Buaba and Abdollah Homaifar, "Optimization of Image Fusion Using Genetic Algorithms and Discrete Wavelet Transform,", Radar signal and image processing, Vol 10, IEEE, 2010.

2. Jun-Ying Gan, Jun-Feng Liu, "Fusion and Recognition of Face and Iris Feature based on Wavelet feature and KFDA,", Proceedings of the 2009 International conference on Wavelet analysis and pattern recognition, boarding, IEEE 12-15 July 2009.

3. S.Arun vivek,J.Araind,S.Valarmathy," Feature Extraction for multimodal biometric and study of fusion using Gaussion Mixture Model,", Proceedings of the international Conference on pattern recognition, Informatics and medical engineering, March 21-21, IEEE, 2012.

4. Mrs.D.Mathivadhani,Dr.C.Meena," Biometric based authentication using Wavelets and visual cryptography,"IEEE-International Conference on recent trends in information technology,ICRTIT, June 3-5, 2011.

5. Jun-Ying Gan,Jian-Hu Gao,Jun-Feng Liu,"Research on Face and Iris feature recognition based on 2DDCT and Kernel Fisher Discriminant Analysis," ,Proceedings of the 2008 International conference on Wavelet analysis and pattern recognition, Hong Kong,30-31 Aug 2008.

6. De-Song Wang,Jian-Ping Li,Yue-HaoYan,"A novel authentication scheme of the DRM system based on multimodal biometric verification and watermarking technique,"IEEE,2008.

7. Ashish Mishra,"Multimodal Biometrics it is:Need for Future System," International journal of computer applications, vol-3-No.4, June 2010.
8. Nick Bartlow,Nathan Kalka,Bjan Cukic and Arun Ross,'Protecting Iris images Through Asymmetric Digital Watermarking”,IEEE,2007.

9. Jie Lin,Jian-Ping Li,Hui Lin,Ji Ming,"'Robust person identification with Face And Iris by modified PUM method,'IEEE,2009.

10. Linlinshen, zhen ji, yuwen li and li bai "Coding Gabor features for multi-modal biometrics Chinese conference on pattern recognition, 2010 IEEE.

11. Sheetalchaudhary, RajendarNath "A fusion mutimodal Biometric Recognition system based on palmprint, fingerprint \& face" International conference on advances in recent technologies in communication and computing,PP.596-600, 2009 IEEE.

12. M.I.Ahmad, w.l.woo,s.s. Dlay“ Multimodal biometric fusion at feature level: face \& palmprint" International conference on symposium ,PP.801-805, 2010 IEEE .

13. Gemma Piella," New quality measures for image fusion," Polytechnical University of Catalonia (UPC), 08034 Barcelona,Spain.

14. Deepak Kumar Sahu1, M.P.Parsai ,'Different Image Fusion Techniques -A Critical Review", International Journal of Modern Engineering Research (IJMER), Vol. 2, Issue. 5, Sep.-Oct. 2012 pp- 4298-4301, ISSN: 2249-6645.

15. Manjusha Deshmukh, Udhav Bhosale,'Image Fusion and Image Quality Assessment of Fused Images", International Journal of Image Processing (IJIP), Volume (4): Issue (5), pg 484508.

16. S. Zebhi, M. R. Aghabozorgi Sahaf, and M. T. Sadeghi,"Image fusion using PCA in CS domain", Signal \& Image Processing : An International Journal (SIPIJ) Vol.3, No.4, August 2012. 